\title{
Transgenic mice as a model to study the role of TGF- $\beta$-related molecules in hair follicles
}

\author{
Manfred Blessing, ${ }^{1,5}$ Lillian B. Nanney, ${ }^{1,2,4}$ Lloyd E. King, ${ }^{3,4}$ C. Michael Jones, ${ }^{1}$ and \\ Brigid L.M. Hogan ${ }^{1}$ \\ ${ }^{1}$ Department of Cell Biology, ${ }^{2}$ Department of Plastic Surgery, ${ }^{3}$ Department of Medicine (Division of Dermatology), and \\ ${ }^{4}$ Department of Veterans Affairs, Vanderbilt University School of Medicine, Nashville, Tennessee 37232 USA
}

\begin{abstract}
There is increasing evidence that members of the TGF- $\beta$ superfamily are important regulators of epithelial growth and differentiation in vivo. Here, transgenic mice have been used to study the role of the TGF- $\beta$-related growth factors BMP-2 and BMP-4 in hair and whisker development. In the mature hair follicle, BMP-2 transcripts are normally seen only in precortex cells at the base of the hair shaft. In the transgenic mice reported here, BMP-4, a closely related molecule, has been ectopically expressed in the outer root sheath of hair and whisker follicles using an expression vector based on the bovine cytokeratin IV ${ }^{\star}$ promoter. In response to transgene expression, both outer root sheath cells below the stem cell compartment and hair matrix cells around the dermal papilla cease proliferation. In addition, the expression pattern of cytokeratin markers is disturbed in some transgenic hair follicles. These results support a model in which members of the TGF- $\beta$ superfamily play an active role in the inhibition of cell proliferation and the onset of expression of trichocyte-specific genes that take place when cells leave the matrix of the follicle and differentiate into shaft cells.
\end{abstract}

[Key Words: Transgenic mice; bone morphogenetic proteins; TGF- $\beta$, hair follicle; epidermis; cytokeratins; cytokeratin promoters]

Received September 28, 1992; revised version accepted December 14, 1992.

The development of the mammalian hair follicle is a complex and dynamic process that provides an attractive model for studying proliferation, differentiation, and cellular interactions during tissue morphogenesis (Hardy 1992). Both hair and whiskers develop from a simple mesenchymal condensation stage (Fig. 1a), through the formation of an ectodermal placode and peg (Fig. 1b), to a fully differentiated, actively growing hair follicle (Fig. 1c). The mature follicle is composed of several concentric layers of epithelial cells and a cluster of specialized mesenchymal cells known as the dermal papilla. In addition, most hair follicles of mammals undergo a growth cycle (Dry 1926): Anagen is the phase of active proliferation and morphogenesis, catagen marks the cessation of hair production and the conversion of the bulb region to an anchoring club (Straile et al. 1961), and telogen describes the resting phase. In vivo studies have shown that the stem cells of the follicle are located in a specialized part of the outer root sheath known as the bulge region (Cotsarelis et al. 1990). During the extension phase (anagen; Fig. 1c), descendants of these stem cells proliferate and differentiate into outer root sheath cells

${ }^{5}$ Corresponding author. that express biochemical markers similar to those seen in interfollicular epidermis for example, epidermal cytokeratins 5, 14, and 17 and the cytokeratin pair 6 and 16 typical for hyperproliferative keratinocytes (Heid et al. 1988a,b; Stoler et al. 1988; Kopan and Fuchs 1989). Subsequently, at the base of the follicle they give rise to rapidly proliferating matrix cells that down-regulate the epidermal cytokeratins and concomitantly switch on a different set of trichocytic cytokeratins (Lynch et al. 1986; Heid et al. 1988a,b; Moll et al. 1988; Powell et al. 1991). Finally, as the cells lose contact with the dermal papilla and move upward, their proliferative potential is lost and they begin to express trichocytic markers at high levels (Heid et al. 1988a,b; Powell et al. 1991).

Despite accumulating data regarding the differential expression of polypeptide growth factors and their receptors in hair follicles (for review, see Hardy 1992), little is known about the precise function of these molecules in either the growth cycle or the differentiation processes described above. Of particular interest are the expression patterns of BMP-2 and BMP-4 (bone morphogenetic proteins 2 and 4 ), members of the TGF- $\beta$ superfamily of polypeptide-signaling molecules (for review, see Lyons et al. 1991). During development, BMP-4 is only transiently expressed in the mesenchymal condensations preceding 


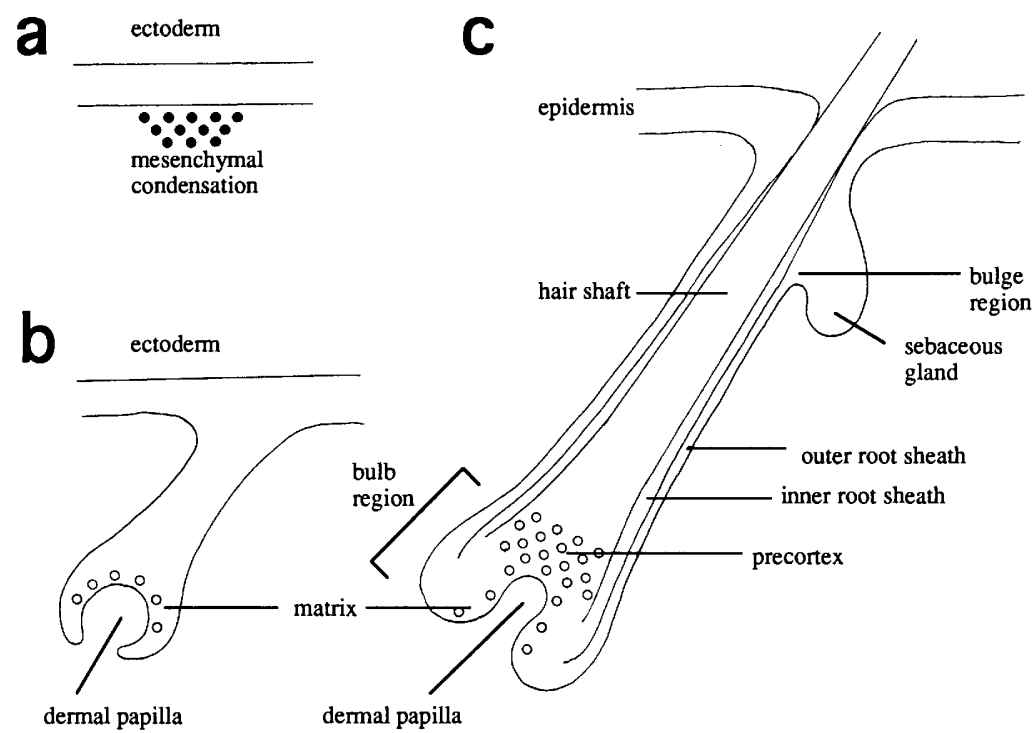

Figure 1. Diagram illustrating the expression patterns of BMP-2 transcripts $(O)$ and BMP-4 transcripts (O) at different stages of hair follicle development: At early stages preceding the thickening and invagination of the ectoderm, BMP-4 is transiently expressed in the mesenchymal condensations $(a)$. In more advanced follicles, BMP-2 is expressed in matrix cells in direct contact with the dermal papilla $(b)$. In mature follicles, moderate BMP-2 expression is seen in basal matrix cells and strong expression is seen in the precortex cells $|c|$. hair follicle formation (Fig. 1a; Jones et al. 1991). BMP-2 is first seen in the epidermal placode (Lyons et al. 1990). Subsequently, as the different layers of the hair follicles develop, expression of BMP-2 becomes restricted to the matrix cells directly in contact with the dermal papilla and the precortex cells (Fig. 1b). In the mature whisker and hair follicle, BMP-2 is expressed exclusively at low levels in the basal cells of the hair matrix surrounding the dermal papilla and at high levels in the cells that have already detached from the dermal papilla to form the base of the shaft (Fig. 1c; Lyons et al. 1990). Therefore, the expression pattern of BMP-2 in mature follicles coincides with the cessation of cell proliferation and the onset of the expression of trichocytic markers. Because several TGF- $\beta$ proteins have been shown to inhibit keratinocyte proliferation (for review, see Fuchs 1990), we have assumed as a working hypothesis that BMP-2 triggers the withdrawal of precortex cells from the cell cycle in an autocrine manner. To investigate this possibility we set out to generate transgenic mice with an expanded domain of BMP-2 expression encompassing the outer root sheath. In addition, we generated transgenic mice expressing the closely related protein BMP- 4 in the outer root sheath. BMP-2 and BMP-4 display $86 \%$ sequence identity at the protein level in the mature carboxy-terminal region (Wozney et al. 1988). They have been shown to mediate identical biological effects in both cartilage and bone induction (Wozney et al. 1988) and the specification of posterior mesoderm in Xenopus development (Dale et al. 1992; Jones et al. 1992; C.M. Jones, unpubl.). This indicates that under some circumstances these two molecules are able to substitute for one another and to evoke similar biological responses.

The expression vector used here is based on the promoter region of the bovine cytokeratin gene $\mathrm{IV}^{*}$, which is the orthologous bovine gene to the human gene for cytokeratin 6 (Blessing et al. 1987, 1989). This gene is expressed in squamous and glandular epithelia (e.g., na- sal epithelium, oral mucosa) and outer root sheath cells of hair follicles (Moll et al. 1982; Heid et al. 1988a,b; Stoler et al. 1988; Kopan and Fuchs 1989). It is not expressed in interfollicular epidermis but can be induced by stimuli that lead to hyperproliferation (e.g., wounding and stripping; for references, see Fuchs et al. 1988; Galvin et al. 1989|. BMP-2 and BMP-4 cDNAs coupled to the cytokeratin $\mathrm{IV}^{\star}$ promoter were used to generate transgenic mice. We obtained only one BMP-2 transgenic founder animal, which was normal and did not express the transgene. However, we obtained cytokeratin $\mathrm{IV}^{*}-$ BMP-4 transgenic mice, which show a complete deficiency of hair growth after the first growth cycle, leading to progressive balding. In addition, the transgenic whiskers are underdeveloped and produce only rudimentary shafts, if any at all. BrdU-labeling experiments indicate that this deficiency results from the absence of cell proliferation in the outer root sheath and the matrix cells. In addition, some transgenic hair follicles show an aberrant keratinization pattern. These results suggest that one function of BMP-2 in the normal hair follicle is the termination of proliferative activity as the cells leave the matrix of the hair bulb and a second likely function is to turn on markers typical for shaft differentiation.

\section{Results}

\section{Phenotypes of transgenic mice}

Despite repeated attempts we have obtained up to now only one transgenic mouse carrying the cytokeratin IV * BMP-2 construct (Fig. 2) from a total of $\sim 220$ injected and transferred eggs. The founder transmitted the transgene to its offspring, but this line showed no obvious phenotype. By in situ hybridization we did not detect expression of the human BMP-2 cDNA in this line. We therefore believe that the embryonic expression of BMP-2 under the control of the cytokeratin $\mathrm{IV}^{\star}$ promoter 
Figure 2. Construction of the cytokeratin III/IV* minilocus expression vector. The genomic organization of the cytokeratin genes III and IV* is shown at the top. Exons are represented by rectangles. Hatched rectangles denote exons that have been deleted in the cytokeratin III/ $/ \mathrm{IV}^{\star}$ minilocus expression vector (bottom). The exon and intron sizes are approximate values. Solid horizontal lines correspond to bovine genomic DNA sequences; the broken line corresponds to the pBR322 vector sequences. To express cDNAs under the control of the cytokeratin III promoter, they have to be inserted into the SalI site. For expression under the control of the cytokeratin $\mathrm{IV}^{\star}$ promoter,

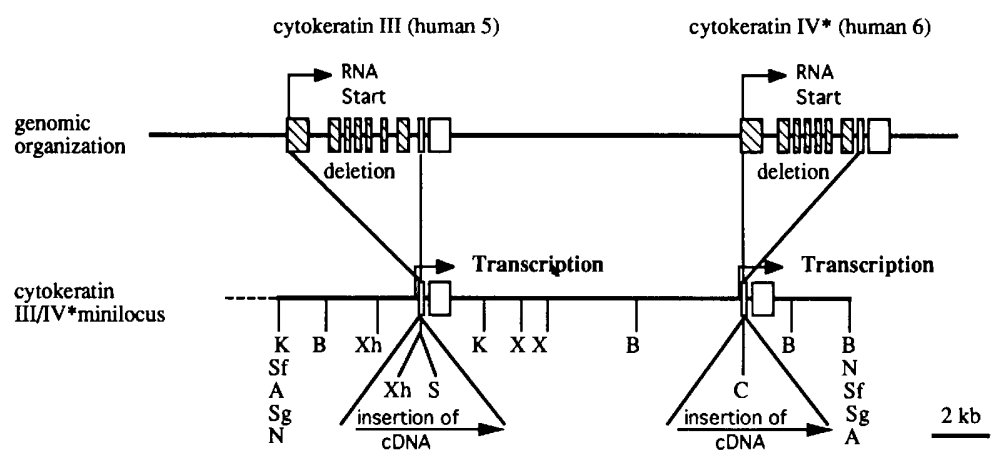
they have to be inserted into the ClaI site. In both cases, the expression vector provides endogenous splice and poly(A) addition sites. We obtained the constructs cytokeratin IV*-BMP-2 and cytokeratin IV*-BMP-4 by inserting human cDNAs for BMP-2 and BMP-4 into the ClaI site of this vector. Restriction enzymes: (A) AatII; (B) BamHI; (C) ClaI; (K) KpnI; (N) NotI; (P) PacI; (S) SalI; (Sf) SfiI; (Sg) SgrAI; (X) SmaI; (Xh) XhoI.

causes prenatal death due to transgene expression in certain epithelia (see below and Discussion).

In contrast, we have obtained a total of 13 transgenic mice carrying the cytokeratin IV*-BMP-4 construct (Fig. 2) from a total of 200 injected eggs. This frequency of $<10 \%$ transgenic offspring differs significantly from the frequency of $\sim 30 \%$ that we usually obtain using other DNA constructs (e.g., BMP-4 lacZ fusion genes). This finding suggests that embryonic expression of BMP-4 under the control of the cytokeratin IV ${ }^{\star}$ promoter leads to the premature death of over two-thirds of the transgenic embryos but nevertheless seems to be more tolerable than ectopic BMP-2 expression. The surviving cytokeratin IV*-BMP-4 transgenic mice could be grouped into three classes according to their phenotype. The most severely affected pups $(n=5$ : TgCK4-1 to TgCK4-5) displayed severe craniofacial malformations, including fusion of the dentary, zygomatic, and maxilla bones and clefting of the secondary palate. In addition, the whiskers (vibrissae) were either completely absent or produced only short stubby shafts. These animals were unable to articulate the jaw and died perinatally. Mice with a moderate phenotype ( $n=6: \mathrm{TgCK} 4-6$ to TgCK4-11) do not display craniofacial malformations but show disturbances both in their body fur (pelage), resulting in balding, and in their whiskers, which produce either no shafts or only short ones. In addition, these animals suffer from a growth retardation of variable severity and only two founder animals have been fertile (TgCK4-6 and TgCK4-11). Two transgenic animals (TgCK4-12 and TgCK4-13) showed no altered phenotype at all. One fertile transgenic founder (TgCK4-6) displaying a moderate phenotype was used to establish a line by mating to $(\mathrm{C} 57 \mathrm{BL} / 6 \times \mathrm{DBA} / 2) \mathrm{F}_{1}$ females. Transgenic offspring show the same abnormalities of the fur and the whiskers as the other five independently derived transgenic animals with this moderate phenotype. In addition, there is variable growth retardation in pups belonging to this line which, in severe cases, leads to premature death.

The abnormality in the fur of these animals is based on the failure of their hair follicles to initiate a second growth cycle, which leaves shed or lost hair unreplen- ished and leads to balding with age (Fig. 3). This absence of regrowth was confirmed by wax-stripping the hair from animals aged 3-5 weeks. Normal and transgenic mice were stripped at their mid-dorsum and observed for a period of up to 3 weeks. The stripped areas in the normal mice showed complete regrowth of the fur within a week, whereas no regrowth was observed in the transgenic animals even after 21 days. To rule out a possible secondary systemic effect owing to BMP-4 misexpression, which might indirectly prevent the regrowth of hair in transgenic animals, a series of skin-grafting experiments were performed. Full thickness samples of stripped skin from normal $(n=3)$ or cytokeratin $\mathrm{IV}^{*}$ BMP-4 transgenic mice of 3-5 weeks of age $(n=6)$ were grafted onto athymic recipients. After 21 days, the grafts derived from normal mice had fully regrown their hairs, whereas the grafts derived from transgenic mice did not show any regrowth of body hairs.

\section{Expression of the human BMP-4 transgene}

To confirm the correct expression of the human BMP-4 cDNA under the control of the cytokeratin $\mathrm{IV}^{\star}$ regulatory elements, in situ hybridization experiments were performed on sections of newborn heads, and stripped or normal dorsal epidermis of mice aged 3-5 weeks. Strong expression of the transgene was seen in the nasal epithelium and weaker expression in the oral mucosa and

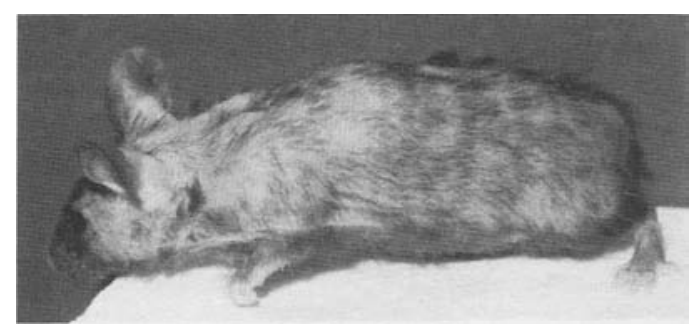

Figure 3. Photograph of the moderately affected transgenic founder TgCK4-10 at 15 months of age, lacking whiskers and suffering from a substantial loss of its fur. 
whisker follicles in newborn animals displaying a severe phenotype (TgCK4-1; data not shown). In animals derived from the moderately affected founder TgCK4-6, expression was weak in the nasal epithelium and very weak in the oral mucosa (data not shown). In the whisker follicles, expression was seen in root sheath cells (Fig. $4 a, b)$. In the epidermis of these animals transgene expression was detected at moderate levels in the outer root sheath of the hair follicles but not in interfollicular epidermis (Fig. 4c,d). However, after stripping, the transgene was expressed at high levels in the interfollicular epidermis and the follicular expression was also greatly elevated (Fig. 4e,f). Similar to the expression in the whisker follicles, human BMP-4 RNA was abundant in the outer root sheath (Fig. $4 \mathrm{c}-\mathrm{f}$ ).

\section{Proliferation in transgenic hair follicles and epidermis}

DNA synthesis in epidermis and hair follicles of normal and cytokeratin $\mathrm{IV}^{*}-\mathrm{BMP}-4$ transgenic mice (offspring of TgCK4-6) was studied by BrdU-labeling experiments. Proliferation in normal, anagen hair follicles ( 7 days after wax-stripping) and epidermis is especially frequent in the matrix cells of the hair bulb region (Fig. 5a, arrowheads) but is also observed in the outer root sheath and the basal cells of the interfollicular epidermis (Fig. 5c, arrowheads). In contrast, cell proliferation is completely absent in the bulb region and the lower part of the outer root sheath of transgenic hair follicles as seen in Figure $5, \mathrm{~b}$ and $\mathrm{d}$. However, in the same transgenic animal, proliferation does occur in basal cells of interfollicular epidermis and in outer root sheath cells located above the entrance point of the sebaceous gland (Fig. 5 b,d, arrowheads). These differences in the distribution of proliferating cells between normal and transgenic skin was also seen in grafted skin samples (not shown). A similar pattern of BrdU incorporation is seen in the whisker follicles. In normal whiskers, proliferation occurs in the matrix cells (Fig. 5e, arrowheads) and the outer root sheath of the mid-region (Fig. 5g, arrowheads). Both regions are inactive in transgenic whiskers (Fig 5f,h).

\section{Morphology of transgenic skin and hair}

The morphology of hairs and whiskers of cytokeratin IV*-BMP-4 transgenic mice, as well as their skin, differs grossly from the morphology of normal hair and skin [Figs. $5(\mathrm{i}-\mathrm{p})$ and 6]. In contrast to the organized, ladderlike pigmentation and even pattern of keratinization of normal body hairs (Fig. 5i), transgenic hairs show irregular pigmentation and keratinization (Fig. 5k) and are generally crooked (Fig. 51). Occasionally, we observe inflammatory processes accompanied by lymphocyte infiltration (Fig. 5j, arrows). As shown in Figure 5m, normal whiskers display concentric layers of shaft and root sheaths cells surrounded by a blood sinus and a connective tissue sheet. Again, the cytokeratin IV IV $^{*}$ BMP-4 transgenic counterparts are highly irregular in shape, with degenerated layers of root sheaths cells and tiny shafts (Fig. 5n,o). In some areas the whiskers do not de- velop at all to a size comparable to normal whiskers (Fig. 5p). Skin from normal (Fig 6a,c,e) and cytokeratin IV*BMP-4 transgenic mice (Fig. $6 \mathrm{~b}, \mathrm{~d}, \mathrm{f}, \mathrm{g}$ ) differ both in their epithelial and connective tissue constituents. The overall number of hair follicles is comparable in normal and transgenic skin (Fig. 6a-d), but the transgenic hair follicles are much smaller than their normal counterparts (Fig. 6e,f). As a result of wax stripping, normal epidermis thickens within 2 days owing to hyperproliferation in the basal cells (data not shown). Its thickness reverts to normal after $\sim 5$ days (Fig. 6e). This response is delayed in the transgenic skin. We did not observe thickening 2 days after wax-stripping (data not shown) but found a considerable increase in thickness after 5 days (Fig $6 \mathrm{~g}$ ). In addition, we found a remarkable difference in dermal thickness between normal and transgenic mice, with the transgenic dermis being much thinner than normal (Fig. $6 a-d)$.

\section{Expression of hair shaft-specific markers}

We examined normal and transgenic hair follicles for the expression of epidermal-type cytokeratins and hair shaftspecific cytokeratins by indirect immunofluorescence experiments using a broad-range cytokeratin antibody and an antibody specific for the acidic hair keratin Al. Skin samples from the mid-dorsum of normal and cytokeratin IV*-BMP-4 transgenic mice were obtained during the first growth cycle ( 7 days of age) and in telogen phase (4 weeks of age). In addition, samples were obtained 3 and 5 days after wax-stripping. Normal mouse hairs in anagen phase show a distinct expression pattern of hair keratins and epidermal-type keratins. Hair keratins are generally restricted to the shaft of the hair follicle whereas the outer root sheath expresses epidermal-type keratins (Fig. 7a-c). Importantly, hair keratin-specific antibodies never react with outer root sheath cells of normal mice (Fig. 7c, arrowhead). Generally, anagen-phase hair follicles of cytokeratin IV*-BMP-4 transgenic mice are smaller and the reactivity with hair keratin-specific antibodies is reduced but properly restricted to the shaft (not shown). However, besides a disrupted morphology, some transgenic hair follicles $(<10 \%)$ show a completely aberrant expression pattern of hair keratins, where the outer root sheath shows weak reactivity with hair keratin-specific antibodies (Fig. $7 \mathrm{~d}-\mathrm{i}$ ). The specificity of this reaction is demonstrated by the lack of reactivity in the epidermis ( $7 \mathrm{f})$, which is decorated with the broad-range anti-cytokeratin antibody (7e). Three and five days after wax-stripping, when normal follicles express high levels of hair keratins, transgenic follicles show no reactivity at all (not shown).

\section{Discussion \\ Use of cytokeratin promoters for targeting transgenes to the epidermis}

Several recent studies on ectopic expression of molecules involved in signaling pathways in the epidermis 

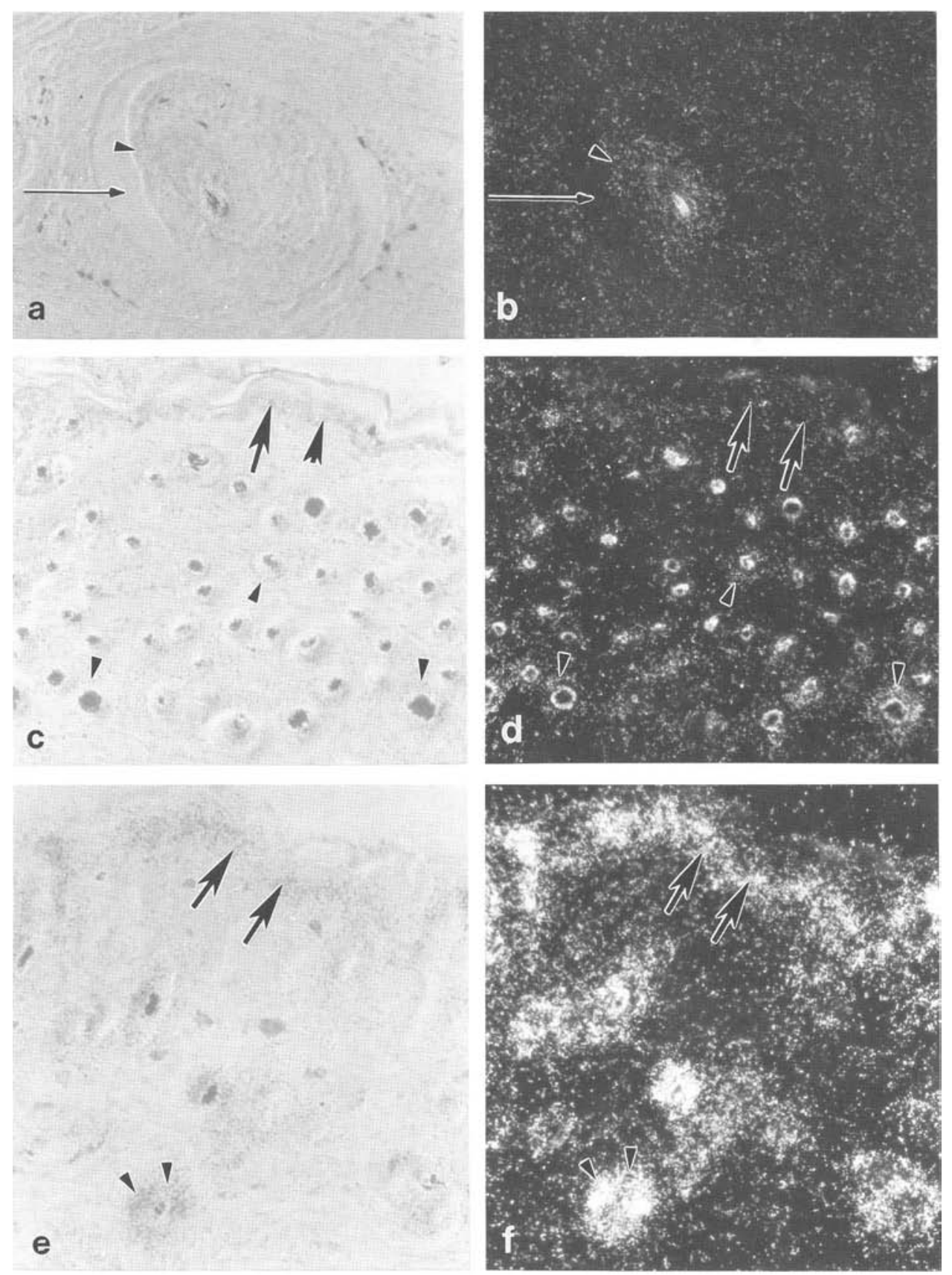

Figure 4. Phase-contrast $(a, c, e)$ and corresponding dark-field illumination $(b, d, f)$ photomicrographs of in situ hybridization experiments using a ${ }^{35}$ S-labeled human BMP-4 cRNA probe. Sections of whiskers $(a, b)$, epidermis $(c, d)$, and epidermis 5 days after waxstripping $(e, f)$ from a 32-day- old cytokeratin IV*-BMP-4 transgenic mouse were processed as described in Materials and methods and exposed for 14 days. Note the weak expression in the epithelial compartment of the whisker follicle $\{a, b$, arrowhead $\}$, which is surrounded by a blood sinus $(a, b$, arrow $)$, and a connective tissue sheath. Normal interfollicular epidermis of transgenic mice does not express the transgene $(c, d$, arrows), whereas the uninduced hair follicles show moderate expression in the outer root sheath $(c, d$, arrowheads). Strong expression is seen after wax-stripping $(e, f)$ in both follicles (arrowheads) and epidermis (arrows). The expression of the transgene is again localized to the outer root sheath of the follicles $\{e, f$, arrowheads $\mid$, whereas the occasionally very strong signal in the center of the follicles $(b)$ is due to light diffraction by highly keratinized and pigmented material. Magnification $(a, b) 86 \times ;(c-f)$ $173 \times$.

have taken advantage of the epithelial specificity and strength of cytokeratin promoters (Bailleul et al. 1990; Vassar and Fuchs 1991; Schönrich et al. 1992; Tinsley et al. 1992; Turksen et al. 1992). Two features of these cytokeratin promoters may affect their general application for targeting transgene expression to the epidermis. First, 


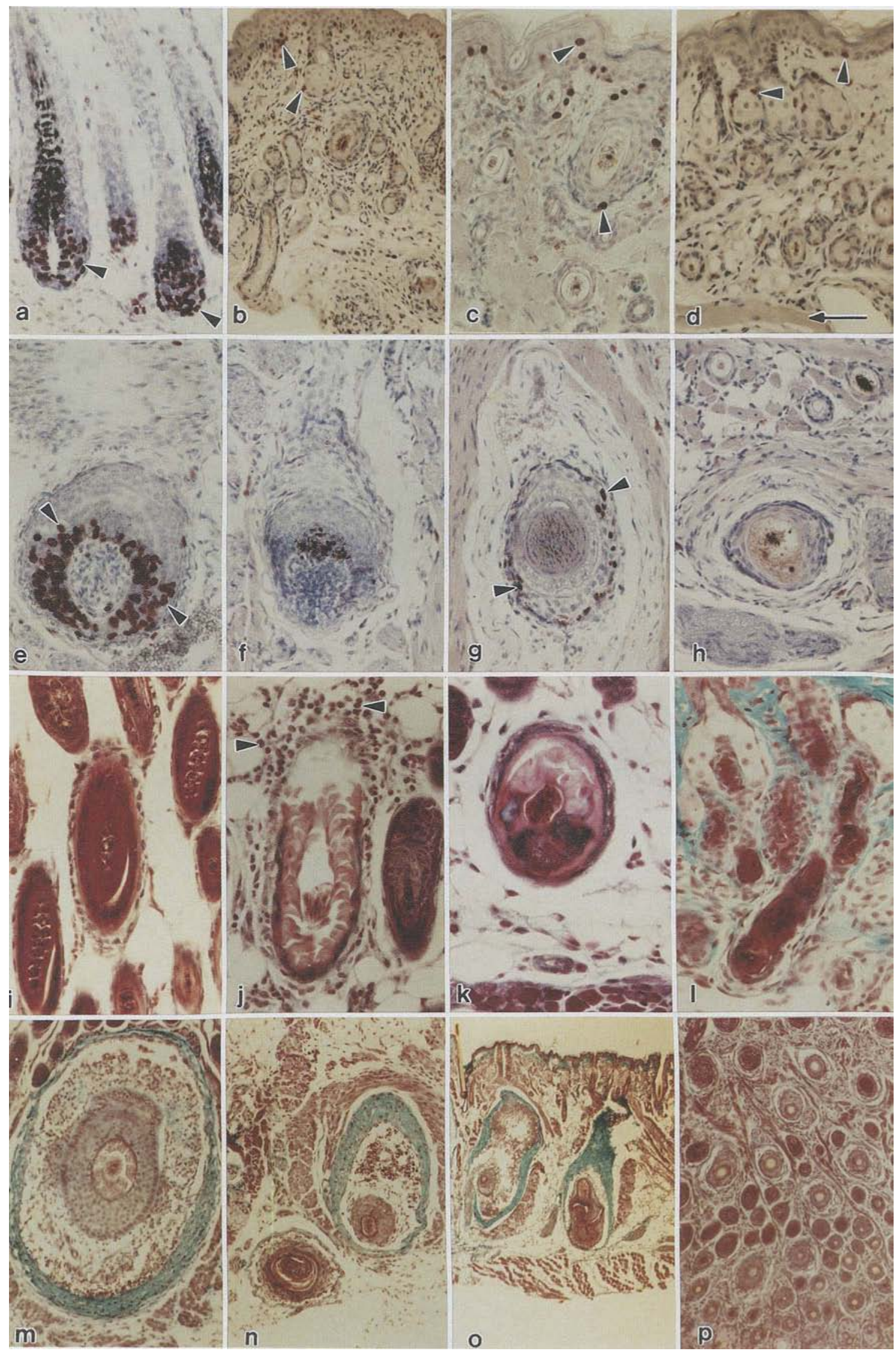

Figure 5. (See following page for legend.) 
the expression of most "epidermal" cytokeratins is not restricted to the epidermis. They are also expressed in a variety of internal squamous or glandular epithelia (Moll et al. 1982; Schönrich et al. 1992). Transgene expression in these epithelia may prevent proper growth of transgenic animals or elicit other pleiotropic effects (Vassar and Fuchs 1991; Turksen et al. 1992). Second, the promoters of cytokeratin genes 10 and 14 are constitutively active in the epidermis as soon as this organ develops during embryogenesis (Dale et al. 1985), which may in some cases result in premature death of transgenic animals or disturb experiments focusing on adult epidermis. Short regions $(<2.2 \mathrm{~kb})$ of the promoter region of cytokeratin $\mathrm{IV}^{\star}$ have been used so far to direct transgene expression to a subpopulation of medullary epithelial cells in the thymus (Schönrich et al. 1992) and to the skin of paws and tails (Tinsley et al. 1992). In the latter case, transgene expression was found in only $20 \%$ of the transgenic founder animals and was generally very weak (Tinsley et al. 1992), indicating a strong dependency of these short promoter fragments on the site of integration. By using a large portion of the bovine cytokeratin $\mathrm{IV}^{\star}$ gene locus we found transgene expression in $\sim 80 \%$ of the surviving transgenic founder animals. We were able to direct transgene expression not only to the outer root sheath of hair follicles but, upon induction, found strong transgene expression in interfollicular dorsal epidermis (see below). The genomic fragments of the cytokeratin $\mathrm{IV}^{\star}$ gene used in our expression vector therefore seem to harbor all of the regulatory elements necessary for correct transgene expression independent of the site of integration, including inducibility. Nevertheless, we have apparently not avoided the pitfall of extra-epidermal expression as we obtained no cytokeratin IV*-BMP-2 transgenic mice expressing the transgene and only a low frequency of surviving cytokeratin IV*-BMP-4 transgenic mice. We therefore believe that cytokeratin $\mathrm{IV}^{*}-$ BMP-2 transgenic mice and many cytokeratin $\mathrm{IV}^{\star}-$ BMP-4 transgenic mice die in utero. The expression patterns of the cytokeratin $\mathrm{IV}^{*}$ transgenes have not been analyzed during early embryogenesis. However, studies on the expression of the human ortholog, cytokeratin 6 , raise the possibility of transgene expression in epithelial cells as early as amnion formation (Regauer et al. 1985). In addition, the severe craniofacial malformations of some of the cytokeratin IV*-BMP-4 transgenic mice are most likely the result of transgene expression in oral mucosa and nasal epithelium at later stages of gestation.

By using the cytokeratin $\mathrm{IV}^{\star}$ promoter, however, we avoid any potential problems of constitutive expression in interfollicular epidermis. This promoter directs transgene expression to the outer root sheath of hair follicles but is inactive in interfollicular epidermis. Nevertheless, it can be strongly induced both in the hair follicle and in interfollicular epidermis by hyperproliferative stimuli (e.g., wax-stripping). Thus, in combination with the technique of skin grafting, which eliminates secondary pleiotropic effects due to transgene expression in internal epithelia, the bovine cytokeratin $\mathrm{IV}^{*}$ promoter may be the promoter of choice for expressing potentially lethal transgenes in the epidermis by grafting transgenic skin from donors or cell culture onto recipients and by inducing transgene expression with hyperproliferative stimuli when suitable.

\section{Effects of BMP-4 misexpression on follicle morphogenesis, cell proliferation, and differentiation}

During hair follicle development, BMP-4 is transiently expressed in mesenchymal condensations preceding the actual follicle bud formation (Jones et al. 1991). BMP-2 is expressed in the cells of the early follicle and later in the precortex but is never seen in outer root sheath cells (Lyons et al. 1990). BMP-2 and BMP-4 are very closely related proteins and have been shown to elicit qualitatively identical effects both in cartilage and bone formation and in the specification of posterior mesoderm in Xenopus (Wozney et al. 1988; Dale et al. 1992; Jones et al. 1992; C.M. Jones, pers. comm.). We therefore assume that BMP-2 and BMP-4 can substitute for one another under some circumstances (i.e., bind to the same receptor|, and our results show that cells in the hair follicle

Figure 5. Distributions of BrdU labeling in the skin and follicles of normal and transgenic mice, carrying the cytokeratin IV*-BMP-4 construct $(a-h)$ and morphological comparisons of normal and transgenic hair follicles and whiskers $(i-p)$. Sections of normal mouse tissues and tissues of transgenic descendants of TgCK4-6 were fixed in paraformaldehyde and stained with Gomori's Trichrome or processed for BrdU incorporation assessment. At 5 days after fur stripping, normal hair follicles show a consistent BrdU labeling of matrix cells within the hair bulb and some labeling of outer root sheath cells $(a, c)$, as demonstrated by the red/brown stain (arrowheads) distinguishable from the black and granular pigment. No DNA synthesis is detected in the matrix cells and the outer root sheath below the entrance point of the sebaceous gland duct (bulge region) in the transgenic counterpart $(b, d)$. The arrow in $d$ points to the dermal-subdermal border demonstrating the reduced dermal thickness, which allows the display of all parts of transgenic hair follicles. In interfollicular epidermis and in the outer root sheath of hair follicles above the bulge region, proliferation takes place both in the normal $|c|$ and transgenic $(b, d)$ tissues at 5 days after fur stripping (arrowheads). In the bulb region of whiskers $(e, f)$ and in the outer root sheath of the mid-region $(g, h)$, numerous labeled nuclei are present only in normal (e.g., arrowheads) but not in transgenic $(f, h)$ follicles, which show only some pigment. Sections of a normal mouse hair follicle at mid-dermal level (i) show a typical pattern of keratinization complete with an organized, ladder-like pigmentation pattern in the cortical region. Defective transgenic hairs $\langle j-I\rangle$ show complete disintegration of internal structures and a surrounding inflammatory infiltrate ( $j$, arrowheads). Hair bulbs and papillae are missing or aberrantly enlarged, differentiation is uneven, and hair follicles are crooked $(k, 1)$. The cross section of a normal whisker $(\mathrm{m})$ shows a connective tissue sheath (green) surrounding the blood sinus and the different layers of the follicle (outer root sheath, inner root sheath, and shaft $)$. Defective $(n, 0)$ and underdeveloped $(p)$ transgenic whiskers show a markedly reduced size and aberrations affecting all components of the follicle. Magnification $(a) 169 \times ;(b) 76 \times ;(c-h) 169 \times ;(i) 169 \times ;(j-1) 352 \times ;(m-0) 55 \times ;(p) 109 \times$. 

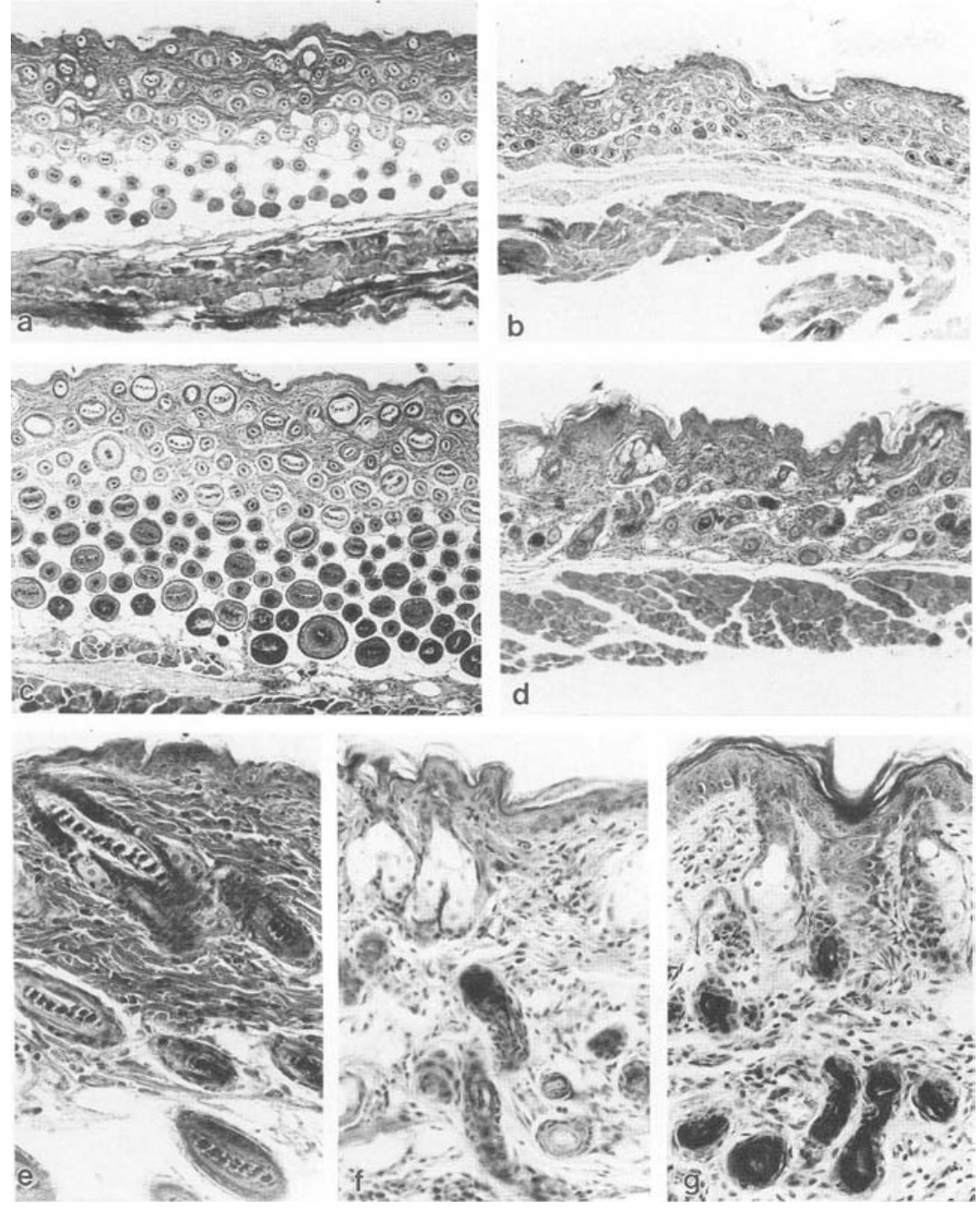

Figure 6. Morphological characterization of normal $(a, c, e)$ and transgenic $(b, d, f, g)$ dorsal mouse skin 5 days after $(c, d, e, g)$ and without wax-stripping $(a, b, f)$. Note thickened epidermis in the stripped transgenic tissue $(d, g)$, defective hair follicles $(f, g)$, and the relatively thin dermis $(b, d, f)$ of the transgenic animals. Magnification $(a-d) 38 \times$; $(e-g) 120 \times$. respond to ectopically expressed BMP-4 in a way that we predicted for ectopic BMP-2 expression. It is therefore most likely that BMP-4 mimics BMP-2 in its effects on outer root sheath cells rather than evoking an unrelated response.

Transgenic animals show a defect in their fur and whiskers. The hair follicles were unable to enter a second growth cycle after the first one was completed, which resulted in progressive balding. This phenotype is quite distinct from the cyclic hair loss reported for a hair keratin-overexpressing transgenic mouse that was able to regrow lost hair (Powell and Rogers 1990). The morphology of the cytokeratin $I^{*}-\mathrm{BMP}-4$ transgenic hair follicles is grossly aberrant. Their size is greatly reduced and their hair production is poor. Interestingly, the dermis of these animals is also affected. It is much thinner than normal dermis, and these animals are generally lean and growth retarded. These observations are similar to the findings with the cytokeratin TGF- $\alpha$ transgenic mice (Vassar and Fuchs 1991), as well as with the cytokeratin IL6 transgenic mice (Turksen et al. 1992), suggesting a generalized pleiotropic effect owing to transgene expres- sion in glandular epithelia as discussed previously. However, the dermis of the moderately affected transgenic mice did not show any signs of ectopic cartilage formation as one might have expected from the powerful boneinducing potentials of BMP-2 and BMP-4 in the rat implantation assay (Wozney et al. 1988). Neither was there any hyperproliferative response in dermal fibroblasts as one might have expected from the fact that BMP-2 and BMP-4 are TGF- $\beta$-related molecules (Massague 1987; Lyons et al. 1991). These differences between physical application of bone morphogenetic proteins by slow release carrier as in the rat implantation assay and delivery through transgene expression in the outer root sheath can be best explained by assuming that active protein derived from the transgene does not reach dermal cells.

BrdU-labeling experiments showed that DNA synthesis was completely shut off in outer root sheath and matrix cells of cytokeratin IV *-BMP-4 transgenic hair follicles below the stem cell compartment in the bulge region. However, DNA synthesis occurs above that level both in outer root sheath cells and basal cells of the epidermis. BMP-4 therefore inhibits proliferation of epi- 
Figure 7. Double label immunostaining of $5-\mu \mathrm{m}$ sections of normal anagen $(a-c \mid$, and cytokeratin $\mathrm{IV}^{*}-\mathrm{BMP}-4$ transgenic $(d-i)$ hair follicles in anagen $(d-f)$ and telogen $(g-$ i) phase. Phase-contrast photomicrographs $\langle a, d, g)_{\text {; }}$ photomicrographs of epifluorescence obtained with a broad-range guinea pig keratin antibody detected with an FITC-conjugated donkey anti-guinea pig antibody $(b, e, h)$, and photomicrographs of epifluorescence obtained with a rabbit acidic A1 hair keratin specific antibody detected with an LRSC-conjugated donkey anti-rabbit antibody $(c, f, i)$. Note the specific reactivity of the hair keratin-specific antibody with the shaft region of the normal hair follicle and the absence of reactivity in the outer root sheath (c, arrowheads), which is decorated with the broad-range keratin antibody $(b$, arrowheads). Some transgenic hair follicles show hair keratinspecific reactivity both in the shaft and weakly in the outer root sheath, which looks abnormally keratinized and degenerated $(e, f, h, i$, arrowheads $)$. Magnification $\mid a-$ c) $109 \times ;(d-f) 79 \times ;(g-i) 109 \times$.
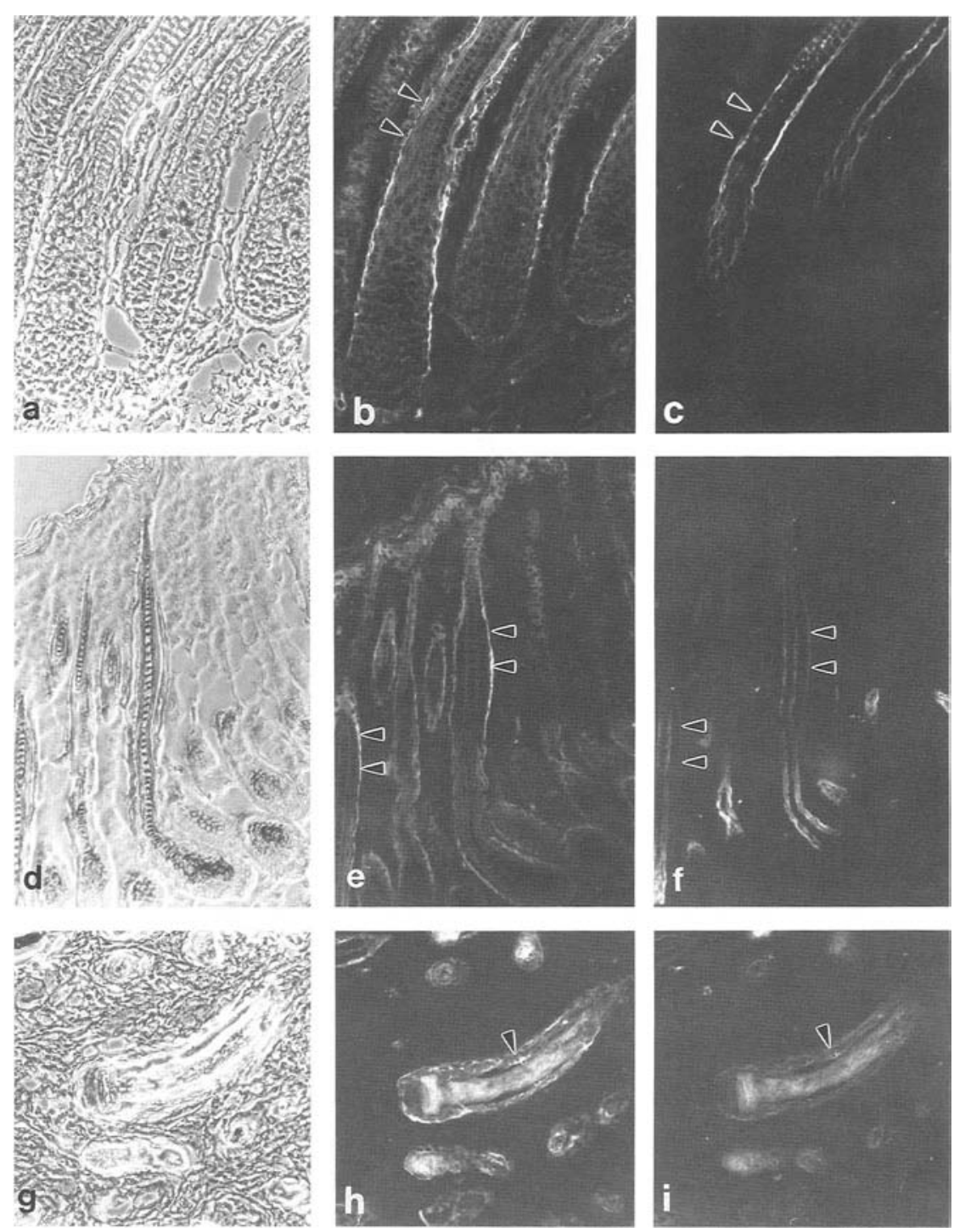

thelial cells that have left the stem cell compartment and are commited to become outer root sheath cells, matrix cells and, finally, terminally differentiate into shaft cells. In the normal hair follicle, cessation of proliferation of matrix cells that have initiated their program of terminal differentiation coincides with the onset of BMP-2 expression (Lyons et al. 1990). Our findings support a model in which one function of BMP-2 in the normal hair follicle is the termination of cell proliferation as matrix cells enter the precortex region in an autocrine manner. According to this model outer root sheath cells that have left the stem cell compartment toward the epidermis are not inhibited by BMP-4 or BMP-2 expression. This suggests that outer root sheath cells above the stem cell compartment are not equivalent to outer root sheath cells beneath the stem cell compartment. After leaving the stem cell compartment, they are either determined to become outer root sheath cells of the upper part of the follicle and eventually keratinocytes, which are not inhibited by BMP- 2 or BMP-4, or to become outer root sheath cells of the lower part of the hair follicle and eventually matrix cells and trichocytes, which cease proliferation in response to BMP- 2 or BMP-4 expression in vivo. Interestingly, during early stages of hair follicle development, BMP-2 RNA is expressed within the epidermal placode (Lyons et al. 1990) without terminating the proliferation of epithelial cells. This suggests that these early follicle cells do not respond to BMP-2 or BMP-4 in the way that they do at later stages, after the formation of the different layers. This might explain why the cytokeratin IV*-BMP-4 transgenic mice develop a relatively decent fur in the first growth cycle.

Another change that coincides with the onset of BMP-2 expression in the mature hair follicle is a switch from epidermal-type cytokeratins to hair-specific cytokeratins (Heid et al. 1988a, b; Stoler et al. 1988; Kopan and Fuchs 1989). To determine a possible role of BMP-2 in this switch, we analyzed normal and transgenic hair follicles in anagen phase for the expression of these markers. Our results suggest that BMP-2 might also be in- 
volved in the onset of expression of trichocytic markers as shown by the transgenic hair follicles with hair keratin reactivity in the outer root sheath. However, these hair follicles represent $<10 \%$ of the total number, with the rest showing no hair keratin expression in the outer root sheath but an overall reduced hair keratin-specific reactivity. This suggests that either the ectopic expression of BMP-4 in most transgenic hair follicles is too weak to turn on trichocytic markers or that under normal circumstances additional factors are required and cells have to go through the matrix to be fully competent for trichocytic differentiation. These results are very similar to the effects of TGF- $\beta$ on interfollicular keratinocytes. This factor is predominantly inhibitory to proliferation and only at very high concentrations leads to abnormal differentiation accompanied by a repression of some markers typical for terminal differentiation (for review, see Fuchs, 1990). In conclusion, the observations presented here, the cessation of proliferation, and the occasional switch of differentiation markers in cytokeratin IV*-BMP-4 transgenic mice, provide strong evidence that members of the BMP gene family are potent signaling molecules in epidermal growth and differentiation, and that most likely BMP-2 is involved both in the cessation of proliferation and in the process of terminal differentiation as cells leave the hair matrix.

\section{Materials and methods}

\section{Construction of expression vector}

The genomic organization of the bovine cytokeratins III and IV* (Lehnert et al. 1984; Blessing et al. 1987) was reconstructed in a pBR322 (Bolivar et al. 1977) derivative that consists of the 1989bp NdeI-AatII fragment containing the origin of replication and the ampicillin resistance gene. A polylinker with the following restriction enzyme recognition sites has been introduced to facilitate the reconstruction of the genomic organization of these cytokeratin genes, as well as the excision of these constructs, to separate them from vector sequences for embryo injection: NdeI-AatII-SgrAI-NotI-SfiI-PacI-KpnI-XhoI-SalI-SacII-SfiINdeI-SgrAI-PacI-AatII. The cytokeratin III/IV* minilocus contains the DNA sequences ranging from $\sim 5.2 \mathrm{~kb}$ upstream of the cytokeratin gene III transcription start site to $\sim 2.7 \mathrm{~kb}$ downstream of the cytokeratin IV ${ }^{\star}$ poly $(A)$ addition site. However, the regions spanning the cap site to the middle of the second to last exon in the case of the cytokeratin gene $\mathrm{IV}^{*}$ and the middle of the 5 '-untranslated region to the middle of the second to last exon in the case of the cytokeratin gene III have been deleted (Fig. 2). Insertion of cDNAs into the SalI site leads to the expression of these cDNAs in basal-type epithelial cells in transgenic mice (data not shown), whereas insertion into the ClaI site leads to transgene expression under the control of the cytokeratin $\mathrm{IV}^{\star}$ promoter, as described in Results. Using 8-mer ClaI linkers we inserted cDNAs for human BMP-2 and human BMP-4 into the ClaI site of this vector to yield the constructs cytokeratin IV*-BMP-2 and cytokeratin IV*-BMP-4, respectively. The human CDNAs for BMP-2 and BMP-4 were generously provided by Dr. John Wozney, Genetics Institute (Cambridge, Massachusetts).

\section{Generation of transgenic mice}

Transgenic mice were generated as described previously (Hogan et al. 1986). The vector sequences were removed from the constructs cytokeratin IV*-BMP-2 and cytokeratin IV IV $^{*}$ BMP-4 by NotI digestion, followed by agarose gel electrophoresis. The $\sim 26-\mathrm{kb}$ insert fragment was excised and purified. Fertilized eggs were obtained from superovulated $(\mathrm{C} 57 \mathrm{BL} / 6 \times \mathrm{DBA} / 2) \mathrm{F}_{1}$ females mated with $\mathrm{C} 57 \mathrm{BL} / 6 \times \mathrm{DBA} / 2 / \mathrm{F}_{1}$ males and injected with DNA solutions at 3-5 $\mathrm{ng} / \mu \mathrm{l}$. The embryos were subsequently transferred into the oviduct of a pseudopregnant female and allowed to come to term. Pups were analyzed for the presence of the transgene by Southern blotting (Southern 1975).

\section{Analysis of transgene expression}

Transgene expression was monitored by in situ hybridization of sections to a ${ }^{35} \mathrm{~S}$-labeled $\mathrm{cRNA}$ probe corresponding to the entire coding region of the human BMP-4 cDNA. No specific hybridization signal was obtained using this probe on sections of normal mouse tissues demonstrating the specificity of this probe for transgene-derived transcripts. The experiments were performed under high stringency conditions essentially as described (Jones et al. 1991).

\section{Animal procedures}

Wax-stripping of mid-dorsal epidermis was performed on anesthetized mice to provide a proliferative stimulus and to synchronize a population of anagen hair follicles. The mid-dorsum was shaved, and coated with molten bee wax, which was peeled off after hardening. Full-thickness skin-grafting experiments were performed as described (Nanney et al. 1992). Wax-stripped or shaved epidermis from the mid-dorsum of transgenic and nontransgenic mice was grafted onto athymic recipients. The hosts were sacrificed 21 days after surgery, and the grafted skin was recovered, and fixed in $4 \%$ paraformaldehyde in phosphate buffered saline (PBS) and prepared for in situ hybridization experiments and BrdU incorporation measurements. BrdU-labeling experiments were performed using the Cell Proliferation Kit (Amersham, Arlington Heights, IL). Mice were injected with 30 $\mu \mathrm{g} / \mathrm{gram}$ body weight of BrdU and sacrificed after a labeling period of $4 \mathrm{hr}$.

\section{Immunostaining}

Indirect immunofluorescence microscopy was performed on sections of normal and transgenic mouse dorsal epidermis. Fresh tissue samples were fixed in methanol/dimethylsulfoxide (DMSO) (4:1), washed several times in methanol, and twice in xylene, and embedded in paraffin for sectioning. Tissue sections $(5 \mu \mathrm{m})$ were dewaxed through xylene, rehydrated by incubation in ethanol twice, once each in $95 \%$ ethanol, $90 \%$ ethanol, $80 \%$ ethanol, $65 \%$ ethanol, and $40 \%$ ethanol, and twice in PBS. Sections were blocked for 10 min with PBS containing $10 \%$ normal donkey serum and incubated with the primary antibodies in PBS containing $10 \%$ normal donkey serum for $45 \mathrm{~min}$. After two washes in PBS for a total of 15 min and blocking with PBS containing $10 \%$ normal donkey serum for $10 \mathrm{~min}$, sections were incubated with fluorochrom-labeled secondary antibodies in PBS with $10 \%$ normal donkey serum for another $45 \mathrm{~min}$. After two final washes in PBS for $15 \mathrm{~min}$ the sections were dipped in distilled water, incubated in ethanol for $1 \mathrm{~min}$, air-dried, and mounted. A keratin antiserum raised in guinea pig against bovine muzzle keratins was purchased from Sigma (St. Louis, $\mathrm{MO}$; cat. no. K4252). It was used at a working dilution of $1 / 60$. Antisera against an acidic (Al) mouse hair keratin (Yu et al. 1991) were kind gifts from Dr. Bertolino and Dr. Chekla (New York University, NY) and were used at a 1/150 dilution. Fluorescein-con- 
jugated donkey anti-guinea pig and Lissamine rhodamineconjugated donkey anti-rabbit antibodies were purchased from Jackson ImmunoResearch Laboratories (West Grove, PA; cat. nos. 706-095-145 and 711-086-132). They were diluted 1/20o. Photomicrographs were taken with a Zeiss Axiophot photomicroscope using Tmax 400 films (Kodak).

\section{Acknowledgments}

We thank N. Cardwell, L. Batts, and L. Hargett for excellent technical assistance and Dr. D. Noden (Cornell University, College of Veterinary Medicine, Ithaca, NY) and Dr. E. Wilkinson (University of Tennessee, Veterinary College,) for their expert advice on the extraepidermal pathology of transgenic mice. We are grateful to D. Chekla and P. Bertolino (New York University Medical School) for the hair keratin-specific antibodies and J. Wozney (Genetics Institute) for providing us with cDNAs for human BMP-2 and BMP-4. This work was supported by funds from the Department of Veterans Affairs to L.B.N. and L.E.K. U.S. Public Health Service grants HD28955 to B.L.M.H., GM40437 to L.B.N., AR-26518 to L.E.K.; and EMBO funding to M.B.

The publication costs of this article were defrayed in part by payment of page charges. This article must therefore be hereby marked "advertisement" in accordance with 18 USC section 1734 solely to indicate this fact.

\section{References}

Bailleul, B., M.A. Surani, S. White, S.C. Barton, K. Brown, M. Blessing, J. Jorcano, and A. Balmain. 1990. Skin hyperkeratosis and papilloma formation in transgenic mice expressing a ras oncogene from a suprabasal keratin promoter. Cell 62: 697-708.

Blessing, M., H. Zentgraf, and J.L. Jorcano. 1987. Differentially expressed bovine cytokeratin genes. Analysis of gene linkage and evolutionary conservation. EMBO J. 6: 567-575.

Blessing, M., J.L. Jorcano, and W.W. Franke. 1989. Enhancer elements directing cell-type-specific expression of cytokeratin genes and changes of the epithelial cytoskeleton by transfection of hybrid cytokeratin genes. EMBO J. 8: 117-126.

Bolivar, F., R.L. Rodriguez, P.J. Greene, M.C. Betlach, H.L. Heyneker, H.W. Boyer, J.H. Crosa, and S. Falkow. 1977. Construction and characterization of new cloning vehicles. II. A multipurpose cloning system. Gene 2: 95-113.

Cotsarelis, G., T.-T. Sun, and R.M. Lavker. 1990. Label-retaining cells reside in the bulge area of pilosebaceous unit: Implications for follicular stem cells, hair cycle, and skin carcinogenesis. Cell 61: 1329-1337.

Dale, B.A., K.A. Holbrook, J.R. Kimball, M. Hoff, and T.-T. Sun. 1985. Expression of epidermal keratins and filaggrin during human fetal skin development. J. Cell Biol. 101: 1257-1269.

Dale, L., G. Howes, B.M.G. Price, and J.C. Smith. 1992. Bone morphogenetic protein 4: A ventralizing factor in early $\mathrm{Xe}$ nopus development. Development 115: 573-585.

Dry, F.W. 1926. The coat of the mouse (Mus musculus). J. Genet. 16: 287-340.

Fuchs, E. 1990. Epidermal differentiation. Curr. Opin. Cell Biol. 2: $1028-1035$.

Fuchs, E., A. Stoler, R. Kopan, and M. Rosenberg. 1988. The differential expression of keratin genes in human epidermal cells. In The biology of wool and hair (ed. G.E. Rogers, P.J. Reis, K.A. Ward, and R.C. Marshall), pp. 287-309. Chapman and Hall, London/New York.

Galvin, S., C. Loomis, M. Manabe, D. Dhouailly, and T.-T. Sun. 1989. The major pathways of keratinocyte differentiation as defined by keratin expression: An overview. Adv. Dermatol. 4: 277-300.

Hardy, M.H. 1992. The secret life of the hair follicle. Trends Genet. 8: 55-60.

Heid, W.W., I. Moll, and W.W. Franke. 1988a. Patterns of expression of trichocytic and epithelial cytokeratins in mammalian tissues. I. Human and bovine hair follicles. Differentiation 37: 137-157.

- 1988b. Patterns of expression of trichocytic and epithelial cytokeratins in mammalian tissues. II. Concomitant and mutually exclusive synthesis of trichocytic and epithelial cytokeratins in diverse human and bovine tissues (hair follicle, nail bed and matrix, lingual papilla, thymic reticulum). Differentiation 37: 215-230.

Hogan, B.L.M., F. Costatini, and E. Lacy. 1986. Manipulating the mouse embryo. A laboratory manual. Cold Spring Harbor Laboratory. Cold Spring Harbor, New York.

Jones, C.M., K.M. Lyons, and B.L.M. Hogan. 1991. Involvement of Bone Morphogenetic Protein-4 (BMP-4) and Vgr-1 in morphogenesis and neurogenesis in the mouse. Development 111: $531-542$.

Jones, C.M., K.M. Lyons, P.M. Lappan, C.V.E. Wright, and B.L.M. Hogan. 1992. DVR-4 (Bone Morphogenetic Protein-4) as a posterior-ventralizing factor in Xenopus mesoderm induction. Development 115: 639-647.

Kopan, R. and E. Fuchs. 1989. A new look into an old problem: keratins as tools to investigate determination, morphogenesis, and differentiation in skin. Genes \& Dev. 3: 1-15.

Lehnert, M.E., J.L. Jorcano, H. Zentgraf, M. Blessing, J.K. Franz, and W.W. Franke. 1984. Characterization of bovine keratin genes: Similarities of exon patterns in genes coding for different keratins. EMBO J. 3: 3279-3287.

Lyons, K.M., R.W. Pelton, and B.L.M. Hogan. 1990. Organogenesis and pattern formation in the mouse: RNA distribution patterns suggest a role for Bone Morphogenetic Protein-2A (BMP-2A). Development 109: 833-844.

Lyons, K.M., C.M. Jones, and B.L.M. Hogan. 1991. The DVR gene family in embryonic development. Trends Genet. 7: 408-412.

Lynch, M.H., W.M. O'Guin, C. Hardy, L. Mak, and T.-T. Sun. 1986. Acidic and basic hair/nail ("Hard") keratins: Their colocalization in upper cortical and cuticle cells of the human hair follicle and their relationship to "soft" keratins. J. Cell Biol. 103: 2593-2606.

Massague, J. 1987. The TGF- $\beta$ family of growth and differentiation factors. Cell 49: 437-438.

Moll, R., W. Franke, D. Schiller, B. Geiger, and R. Krepler. 1982. The catalog of human cytokeratins: Patterns of expression in normal epithelia, tumors and cultured cells. Cell 31: 11-24.

Moll, I., H.W. Heid, W.W. Franke, and R. Moll. 1988. Patterns of expression of trichocytic and epithelial cytokeratins in mammalian tissues. III. Hair and nail formation during human fetal development. Differentiation 39: 167-184.

Nanney, L.B., R.A. Yates, and L.E. King Jr. 1992. Modulation of epidermal growth factor receptors in psoriatic lesions during treatment with topical EGF. I. Invest. Dermatol. 98: 296301.

Powell, B.C. and G.E. Rogers. 1990. Cyclic hair-loss and regrowth in transgenic mice overexpressing an intermediate filament gene. $E M B O$ I. 9: 1485-1493.

Powell, B.C., A. Nesci, and G.E. Rogers. 1991. Regulation of keratin expression in hair follicle differentiation. Ann. N.Y. Acad Sci. 642: 1-20.

Regauer, S., W.W. Franke, and I. Virtanen. 1985. Intermediate filament cytoskeleton of amnion epithelium and cultured amnion epithelial cells: Expression of epidermal cytokera- 
tins in cells of a simple epithelium. J. Cell Biol. 100: 9971009.

Schönrich, G., F. Momburg, G.J. Hämmerling, and B. Arnold. 1992. Anergy induced by thymic medullary epithelium. Eur. J. Immunol. 22: 1687-1691.

Southern, E. 1975. Detection of specific sequences among DNA fragments separated by gel electrophoresis. I. Mol. Biol. 98: 503-517.

Stoler, A., R. Kopan, M. Duvic, and E. Fuchs. 1988. The use of monospecific antibodies and cRNA probes reveals abnormal pathways of differentiation in human epidermal diseases. $I$. Cell Biol. 107: 427-446.

Straile, W.E., H.B. Chase, and C. Arsenault. 1961. Growth and differentiation of hair follicles between periods of growth and quiescence. J. Exp. Zool. 148: 205-222.

Tinsley, J.M., C. Fisher, and P.F. Searle. 1992. Abnormalities of epidermal differentiation associated with expression of the human papillomavirus type 1 early region in transgenic mice. J. Gen. Virol. 73: 1251-1260.

Turksen, K., T. Kupfer, L. Degenstein, I. Williams, and E. Fuchs.1992. Interleukin 6: Insights to its function in skin by overexpression in transgenic mice. Proc. Natl. Acad. Sci. 89: 5068-5072.

Vassar, R. and E. Fuchs. 1991. Transgenic mice provide new insights into the role of TGF- $\alpha$ during epidermal development and differentiation. Genes \& Dev. 5: 714-727.

Wozney, J.M., V. Rosen, A.J. Celeste, L.M. Mitsock, M.J. Whitters, R.W. Kris, R.M. Hewick, and E.A. Wang. 1988. Novel regulators of bone formation: Molecular clones and activities. Science 242: 1528-1534.

Yu, D.-W., S. Yuk-Ying, D.M. Checkla, I.M. Freedberg, T.-T. Sun, and A.P. Bertolino. 1991. Transient expression of mouse hair keratins in transfected HeLa cells: Interactions between "hard" and "soft" keratins. I. Invest. Dermatol. 97: 354-363. 


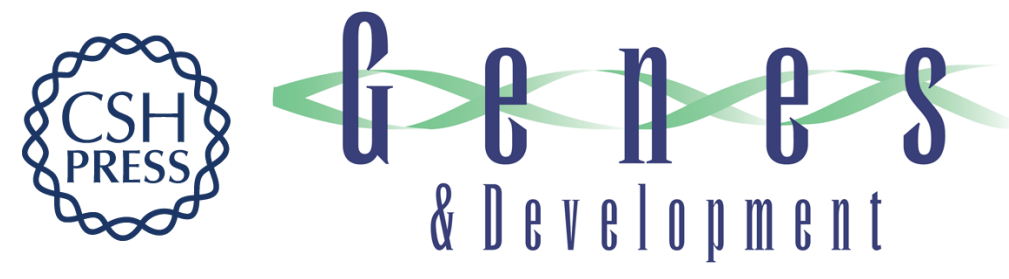

\section{Transgenic mice as a model to study the role of TGF-beta-related molecules in hair follicles.}

M Blessing, L B Nanney, L E King, et al.

Genes Dev. 1993, 7:

Access the most recent version at doi:10.1101/gad.7.2.204

References This article cites 36 articles, 11 of which can be accessed free at:

http://genesdev.cshlp.org/content/7/2/204.full.html\#ref-list-1

License

Email Alerting

Service

Receive free email alerts when new articles cite this article - sign up in the box at the top right corner of the article or click here.

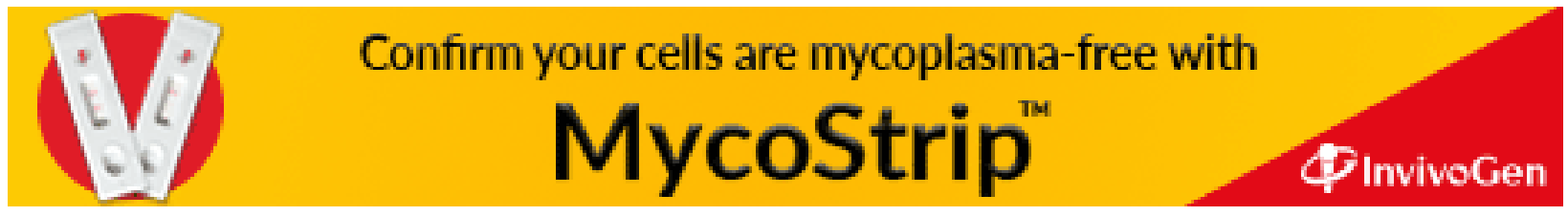

